\title{
Performance Analysis of Decode and Forward Cooperative Relaying over the Generalized-K Channel
}

\author{
Ali Dziri ${ }^{1}$, Michel Terre ${ }^{1}$, Nidal Nasser ${ }^{2}$ \\ ${ }^{1}$ CEDRIC-LAETITIA, Conservatoire National des Arts et Metiers, Paris, France; ${ }^{2}$ College of Engineering, Alfaisal University, \\ Riyadh, KSA. \\ Email: ali.dziri@cnam.fr,michel.terre@cnam.fr, nnasser@alfaisal.edu
}

Received December $29^{\text {th }}, 2012$; revised February $9^{\text {th }}, 2013$; accepted February $23^{\text {rd }}, 2013$

Copyright (C 2013 Ali Dziri et al. This is an open access article distributed under the Creative Commons Attribution License, which permits unrestricted use, distribution, and reproduction in any medium, provided the original work is properly cited.

\begin{abstract}
In this paper, we analyze the performance of cooperative diversity using adaptive Decode and Forward (DF) relaying over independent but non-necessarily identical flat composite fading channels which include multipath fading and shadowing simultaneously. We have considered the transmission of M-QAM modulated signals over Generalized-K channel model which is very versatile and accurately approximates many of the commonly used channel models as Nakagami-m, Log-Normal distributions and the mixture of the two distributions for the composite fading. Using an approximation of the Generalized-K pdf by a Gamma pdf, we derive analytical expressions for the outage probability and the Average Symbol Error Probability (ASEP) of the proposed scenario and analyze their dependence on the channel parameters. We assume in our context that the relay decides independently whether or not to forward the signal to the destination based on the received signal quality. Simulations are provided for 16-QAM modulation for both the outage and the ASEP. The obtained results are discussed and proved to be in good agreement with our theoretical analysis.
\end{abstract}

Keywords: Cooperative; Relay; Generalized-K Distribution; Composite Fading

\section{Introduction}

Multiple-Input Multiple-Output (MIMO) communication schemes have been widely studied to achieve diversity advantages, increased data rate, and interference suppression. However, one of the disadvantages of MIMO systems is the relatively large size needed of a wireless terminal. This is due to the requirement of an antenna spacing equal to half the wavelength to achieve sufficiently uncorrelated signals at the antenna connectors. Recently, it has been shown that cooperative diversity or user cooperation offers spatial diversity when multiple users cooperate to build a virtual antenna array $[1,2]$, benefiting on MIMO advantages. Such a system aims to increase the channel capacity and to improve the link reliability of the wireless communications [2]. To this end, various relaying strategies have been investigated $[3,4]$. We distinguish mainly two techniques: Amplify and Forward (AF) (also known as analog relaying) and Decode and Forward (DF) (also known as digital relaying). In the former strategy, the relay node amplifies the received signal and then retransmits it. Thus, one disadvantage to be mentioned is the increased noise. In the latter strategy, the relay detects and possibly decodes the source message and then regenerates and retransmits it. This technique, is more reliable but it requires more processing compared to AF relaying [5]. The focus of this work is on DF relaying strategy.

To evaluate the system performance, we need to consider the propagation channel characteristics. In our context we consider the wireless channel which poses a severe challenge for reliable high-speed communication due to reflection, diffraction, scattering and absorption. To capture and model all these effects, many scientific research were carried out and they were classified mainly into three fading channel types: path-loss, multipathfading (also known as small-scale fading) and shadowing (also known as large-scale fading).

Multipath fading is relatively fast and it is, therefore, responsible for the short-term signal variations. Depending on the radio propagation environment and the underlying communication scenario, there is a range of statistical multipath fading models available in the literature, such as Rayleigh, Rician, or Nakagami-m distributions [6]. The large-scale fading (shadowing) is due to objects obstructing the propagation path as trees and buildings, 
giving rise to random variations of the received power at a given distance. These fluctuations are experienced on local-mean powers. They are often modeled using a Lognormal distribution [7].

The combination of multipath fading and shadowing effects is usually known as composite fading. A versatile distribution to model the composite fading is the Generalized-K model which demonstrated a superior fit to the measured data over a wide range of physical channel conditions $[8,9]$. Thus, it has attracted a great deal of research interest recently. In [10], the authors have derived the outage probability, the average channel capacity and the ASEP for different modulated signals over the Generalized-K distribution. In [11], they have evaluated the spatial diversity performance over the Generalized-K channel.

Despite the multiplying number of scientific works on the cooperative diversity techniques mentioned above, most of them are limited to the Rayleigh, Rician, Lognormal and Nakagami-m channels. In $[12,13]$, the authors studied the outage and the average error probability of adaptive DF cooperative networks with identical and non-identical Rayleigh fading channels. In $[14,15]$ the authors derived the closed form for the outage probability of an adaptive DF over i.i.d Nakagami-m fading channels. In $[16,17]$, the authors derived the closed form for the outage probability, the ASEP and the average channel capacity based on the MGF expression of the Nakagami-m distribution. In [18], AF cooperative diversity networks have been investigated under Generalized-K distribution. However, for a DF relaying over shadowed-Nakagami channels modeled by Generalized$\mathrm{K}$ distribution, performance analysis has never done according to our best knowledge. Recently, we have evaluated the outage probability [19], and the ASEP for M-QAM modulated signals [20], of a DF relaying technique over a Generalized-K channel. However, a complete performance analysis for different propagation environments conditions is necessary. Thus, a more detailed theoretical analysis will be presented by considering the DF relaying over the Generalized-K channel. The performance metrics under consideration are the outage probability and the ASEP. Different propagation environments will be investigated. The remainder of this paper is organized as follows. In Section 2, we introduce the system model of the DF relaying over the Generalized-K fading model. We have introduced an approximation of the Generalized-K pdf by a Gamma pdf which serves for the outage and the ASEP analysis. The closed form derivations for the outage probability and the ASEP of the non cooperative system (direct link) and the cooperative system are presented in Sections 3 and 4, respectively. Simulation results are given in Sections 5 and 6 concludes this paper.

\section{System and Channel Models}

\subsection{Relay System Model}

We consider a dual-hop cooperative wireless system consisting of $J+2$ wireless nodes: one source node $S$, a set of $J$ relay nodes, $R_{1}, R_{2}, \cdots, R_{J}$, and a destination node $D$. We assume that each node has a single antenna and all $R_{j}$ operate in half-duplex mode. We consider a time-division channel allocation scheme with $J+1$ time slots. In the first time slot, the source broadcasts its message to all the nodes. During the following $J$ time slots, the source stops transmitting and the relays of the decoding set will forward the source information to the destination in a scheduled order. $Q$ denotes the set of indexes of relays which succeed in decoding correctly the source message for a given required SNR threshold, i.e., each relay decides whether to forward the source information or not by comparing the received signal power with a decision SNR threshold. The cardinality of $Q$ is denoted $\bar{J}$. In the considered scenario, we assume that the receivers at the relays and destination have exact channel state information but no transmitter channel state information is available at the source or relays [14].

Let us denote $h_{0}, \tilde{h}_{j}$ and $h_{j}$ as the channel gains corresponding to the direct link, links between the source to the $j$-th relay and the $j$-th relay to the destination, respectively. Moreover, $\tilde{h}_{j}$ and $h_{0}, h_{j}$ are supposed independent but not necessarily identical Generalized-K distributions with parameters $\tilde{m}_{m}^{j}, \tilde{m}_{s}^{j}$ for $j=1, \cdots, J$ and $m_{m}^{j}, m_{s}^{j}$ for $j=0,1, \cdots, J$, respectively. Let $\tilde{\gamma}_{j}$ and $\gamma_{j}$ denote the instantaneous SNR received at the the $j$-th relay and the destination from the $j$-th relay, respectively. Thus,

$$
\tilde{\gamma}_{j}=\left|\tilde{h}_{j}\right|^{2} \frac{E s}{N 0} \text { and } \gamma_{j}=\left|h_{j}\right|^{2} \frac{E s}{N 0}
$$

where $E s$ is the transmit energy signal and $N 0$ represents the single sided power spectral density of the AWGN. The corresponding average SNR are denoted $\overline{\tilde{\gamma}}_{j}$ and $\bar{\gamma}_{j}$. Finally, we denote $\gamma_{D}=\sum_{j=0}^{\bar{J}} \gamma_{j}$ as the contribution of all the relays belonging to the set $Q$ and the direct link using the MRC (Maximum Ratio Combiner) technique.

\subsection{The Generalized-K Fading Channel Model}

The small scale multipath fading is usually modeled using Nakagami-m distribution. The envelope of the transmitted signal over the Nakagami fading, $X$, has a probability density function $p_{X}(x)$ given as follows [21]

$$
p_{X}(x)=\frac{2}{\Gamma\left(m_{m}\right)}\left(\frac{m_{m}}{\Omega}\right)^{m_{m}} x^{2 m_{m}-1} \mathrm{e}^{-\frac{m_{m}}{\Omega} x^{2}}, x>0,
$$


where $m_{m},\left(0.5 \leq m_{m}<+\infty\right)$, is the fading severity parameter called also the Nakagami parameter, $\Omega$, $\left.\Omega=X^{2}\right)$, is the average received power and $\Gamma($.$) is$ the Gamma function.

However, for example in terrestrial and satellite landmobile systems, the link quality is also affected by slow variation of the mean signal level due to the shadowing from terrain, buildings, and trees. Consequently, multipath fading are superimposed on shadowing. Hence, the pdf of the envelope of the received signal $p_{X}(x)$ will be conditioned on the average power $\Omega$ leading to:

$$
p_{X \mid \Omega}(x \mid y)=\frac{2}{\Gamma\left(m_{m}\right)}\left(\frac{m_{m}}{y}\right)^{m_{m}} x^{2 m_{m}-1} \mathrm{e}^{-\frac{m_{m}}{y} x^{2}}, x>0,
$$

Consequently, in combined multipath and shadowing fading, (1) can be rewritten as follows

$$
p_{X}(x)=\int_{0}^{+\infty} p_{X \mid \Omega}(x \mid y) p_{\Omega}(y) \mathrm{d} y, x>0,
$$

where $p_{\Omega}(y)$ is the pdf of the average power which is usually modeled by the Lognormal distribution [21]. To mitigate difficulties to derive closed form of some metric performance measure, using the moment matching method, it has been proposed to use the Gamma distribution to model the average power random variations given as follows

$$
p_{\Omega}(y)=\frac{1}{\Gamma\left(m_{s}\right)}\left(\frac{m_{s}}{\Omega_{0}}\right)^{m_{s}} y^{m_{s}-1} \mathrm{e}^{-\frac{m_{s}}{\Omega_{0}} y}, y \geq 0
$$

where $m_{s}>0$ reflects the shadowing severity and $\Omega_{0}$ is the local mean power.

Consequently, the pdf of the received signal envelope due to the composite fading (shadowing and multipath) leads to the Generalized-K distribution which is given by

$$
\begin{gathered}
p_{X}(x)=\frac{4 \beta^{\frac{m_{m}+m_{s}}{2}}}{\Gamma\left(m_{m}\right) \Gamma\left(m_{s}\right)} x^{m_{m}+m_{s}-1} K_{m_{s}-m_{m}}(2 \sqrt{\beta} x), \\
x \geq 0, \quad m_{m} \geq 0.5, \quad m_{s}>0,
\end{gathered}
$$

where $K_{m_{s}-m_{m}}$ is the modified Bessel function of the second kind and order $m_{s}-m_{m}$ and $\beta=m_{m} m_{s} / \Omega_{0}$.

In the cooperative system, as mentioned in [2], to capture the path-loss effects, we use

$\Omega_{0}=E\left(\left|h_{0}\right|^{2}\right) \sim\left(\frac{1}{d_{S, D}}\right)^{\alpha}, \quad \Omega_{0, j}^{S, R_{j}}=E\left(\left|\tilde{h}_{j}\right|^{2}\right) \sim\left(\frac{1}{d_{S, R_{j}}}\right)^{\alpha}$, $\Omega_{0, j}^{R_{j}, D}=E\left(\left|h_{j}\right|^{2}\right) \sim\left(\frac{1}{d_{R_{j}, D}}\right)^{\alpha}$ where $d_{i, j}$ is the distance between the terminals $i$ and $j$, and $\alpha$ is the pathloss exponent.

Over a Generalized-K fading channel the pdf of the output instantaneous SNR $\gamma$ follows a Generalized-K distribution given by [11]

$$
\begin{aligned}
p_{\gamma}(\gamma)= & \frac{2 \xi^{\frac{m_{m}+m_{s}}{2}}}{\Gamma\left(m_{m}\right) \Gamma\left(m_{s}\right)} \gamma^{\frac{m_{m}+m_{s}}{2}-1} K_{m_{s}-m_{m}}(2 \sqrt{\xi \gamma}), \\
& \gamma \geq 0, \quad m_{m} \geq 0.5, \quad m_{s}>0,
\end{aligned}
$$

where $\xi=\frac{m_{m} m_{s}}{\bar{\gamma}}$ and $\bar{\gamma}$ is the average SNR over the considered channel.

In the next section, we deal with the outage probability and the ASEP closed form derivations. To this end, in the cooperative case, we deal with the cdf of the sum of $\bar{J}+1$ Generalized-K random variables. As pointed in $[22,23]$, the computation of the cdf expression is not straightforward and requires the use of approximations and asymptotic expansions or the use of the numerical inversion of the characteristic function. An alternative approach, to avoid the analytical difficulties, is to consider an approximation of the Generalized-K pdf, given by (6), using the moment matching method by a more tractable pdf [22], as shown in the next subsection.

\subsection{The Approximation of a Generalized-K pdf}

The main goal of this subsection is to give the analytical and main results of the approximation of the Generalized-K pdf by a suitable Gamma pdf. This approximation has been developed in two steps [22]:

- Approximation of the Generalized-K pdf by a Gamma pdf by matching the two first positive moments.

- Introduction of an adjustment factor for the shape and the scale parameters of the approximating Gamma pdf.

If we consider the distribution given by (5), the $n$-th moment of the Generalized-K distribution can be derived as [22]

$$
E\left(X^{n}\right)=\frac{\Gamma\left(m_{m}+n\right) \Gamma\left(m_{s}+n\right)}{\Gamma\left(m_{m}\right) \Gamma\left(m_{s}\right)}\left(\frac{\Omega_{0}}{m_{m} m_{s}}\right)^{n} .
$$

The n-th moment of the approximating Gamma distribution can be expressed as

$$
E\left(X^{n}\right)=\frac{\Gamma(k+n)}{\Gamma(k)} \theta^{n},
$$

where $\theta$ and $k$ denote the scale and shape parameters of the approximating Gamma distribution, respectively.

Matching the two first positive moments of the Generalized-K distribution to those of the approximating Gamma distribution results in

$$
\theta=A F \cdot \Omega_{0}, \quad \text { and } \quad k=1 / A F
$$

where $A F=\frac{1}{m_{m} m_{s}}+\frac{1}{m_{m}}+\frac{1}{m_{s}}$ is the amount of fading 
in the composite fading channel and

$0<A F<A F_{\text {max }}, A F_{\max }$ is determined by the smallest physical values of $m_{m}$ and $m_{s}$. However, this approximation results in a poor fitting in the lower and upper tail regions as compared to the original pdf. In order to bypass this inaccuracy, an adjustable form has been introduced for the scale and shape parameters of the region-wise approximating Gamma pdf for the two first moments. These parameters are given as follows

$$
\tilde{\theta}=(A F-\varepsilon) \Omega_{0}, \quad \tilde{k}=\frac{1}{A F-\varepsilon}, \quad \tilde{\theta}>0, \tilde{k}>0,(10
$$

where the adjustment parameter $\varepsilon$ is chosen so that the pdf (cdf) of the approximating Gamma distribution becomes as close as desired to the pdf (cdf) of the Generalized-K distribution in a certain region of interest. We note that $-A F<\varepsilon<A F$. Since, the relevant practical range of $A F$ is from zero (for non-fading channels) to 8 (for severe multipath fading and shadowing conditions corresponding to $m_{m}=0.5$ and $m_{s}=0.5$ ), the relevant range of the adjustment factor is $-8<\varepsilon<8$.

\subsection{Approximation of the pdf of the Sum of $N$ Independent Generalized-K Random Variables}

As aforementioned in Subsection 2.3, the pdf of the sum of $N$ Generalized-K random variables can be approximated by a Gamma pdf using the adjustable form.

$$
\begin{gathered}
\tilde{\theta}_{N}=\frac{\sum_{i=1}^{N} K_{i} \Omega_{0, i}^{2}+2 \sum_{i=1}^{N} \sum_{j=i+1}^{N}\left(\Omega_{0, i} \Omega_{0, j}\right)}{\sum_{i=1}^{N} \Omega_{0, i}} \\
-\sum_{i=1}^{N}\left(\Omega_{0, i}+\frac{\varepsilon_{i}}{N}\right), \tilde{\theta}_{N}>0, \\
\tilde{k}_{N}=\frac{\sum_{i=1}^{N} \Omega_{0, i}}{\tilde{\theta}_{N}}, \quad \tilde{k}_{N}>0,
\end{gathered}
$$

where $K_{i}=1+\frac{1}{m_{m}^{i}}+\frac{1}{m_{s}^{i}}+\frac{1}{m_{m}^{i} m_{s}^{i}} \cdot m_{m}^{i}$ and $m_{s}^{i}$ are the shape parameters of the $i$-th Generalized-K random variable.

We note that for the i.i.d case, the expressions in (11) and (12) simplify to

$$
\begin{aligned}
& \tilde{\theta}_{N}=(A F-\varepsilon) \Omega_{0}, \quad \tilde{\theta}_{N}>0, \\
& \tilde{k}_{N}=\frac{N}{A F-\varepsilon}, \quad \tilde{k}_{N}>0,
\end{aligned}
$$

An illustration of the choice of the optimal value of the adjustment factor is given in Section 5 .

\section{Performance of the Non Cooperative System (Direct Link)}

In the following, we consider the outage probability and the ASEP as performance system metrics.

\subsection{Outage Probability}

The outage probability represents the probability of unsatisfactory signal reception. It can be defined as the probability that the received SNR is less than a specified SNR threshold value at the destination, denoted $\gamma_{D}^{\text {th }}$ [10]. We consider the direct link composed by the source and the destination node communicating over the composite fading channel. The outage probability denoted $P_{\text {out }}$ is given as [10]

$$
P_{\text {out }}=P\left(\gamma_{0}<\gamma_{D}^{\text {th }}\right)=\int_{0}^{\gamma_{D}^{\text {th }}} p_{\gamma_{0}}(\gamma) \mathrm{d} \gamma .
$$

We note that $p_{\gamma_{0}}(\gamma)$ is given by (6). According to [10], we can derive $P_{\text {out }}$ as follows

$$
\begin{aligned}
P_{\text {out }}= & \pi \csc \left(\pi\left(m_{s}^{0}-m_{m}^{0}\right)\right) \\
& \cdot\left[\frac{\left(\xi \gamma_{D}^{\text {th }}\right)^{m_{m}^{0}}{ }_{1} F_{2}\left(m_{m}^{0} ; 1+m_{m}^{0}-m_{s}^{0} ; m_{m}^{0}+1 ; \xi \gamma_{D}^{\text {th }}\right)}{\Gamma\left(m_{s}^{0}\right) \Gamma\left(1+m_{m}^{0}-m_{s}^{0}\right) \Gamma\left(1+m_{m}^{0}\right)}\right. \\
& \left.-\frac{\left(\xi \gamma_{D}^{t h}\right)^{m_{s}^{0}}{ }_{1} F_{2}\left(m_{s}^{0} ; 1+m_{s}^{0}-m_{m}^{0} ; m_{s}^{0}+1 ; \xi \gamma_{D}^{\text {th }}\right)}{\Gamma\left(m_{m}^{0}\right) \Gamma\left(1+m_{s}^{0}-m_{m}^{0}\right) \Gamma\left(1+m_{s}^{0}\right)}\right],
\end{aligned}
$$

where $\xi=\frac{m_{m}^{0} m_{s}^{0}}{\bar{\gamma}_{0}}, \csc (\cdot)$ is the cosecant function, and ${ }_{p} F_{q}(\because \because \because \cdot)$ is the generalized hyper-geometric function [24].

\subsection{Average Symbol Error Probability}

The ASEP, $P_{s e}$, for modulated signals over the fading channel $h_{0}$ can be obtained by averaging its conditional symbol error probability, $P_{s e}(\gamma)$, under a given SNR $\gamma$ in AWGN over the pdf of the received SNR $p_{\gamma_{0}}(\gamma)$

$$
P_{s e}=\int_{0}^{+\infty} P_{s e}(\gamma) p_{\gamma_{0}}(\gamma) \mathrm{d} \gamma .
$$

In this paper, we consider M-QAM modulated signals over Generalized-K fading channel. Thus, $P_{s e}(\gamma)$ can be expressed as $P_{\text {se }}(\gamma)=\operatorname{Aerfc}(\sqrt{B \gamma})$ [6], where $A=2-2 / \sqrt{M}, B=3 /(2(M-1))$ and $\operatorname{erfc}(x)=\frac{2}{\pi} \int_{x}^{+\infty} \mathrm{e}^{-t^{2}} \mathrm{~d} t$. By considering the same notations above, the closed form of the ASEP for the direct link is given by [10]. 


$$
\begin{aligned}
P_{s e}= & \frac{A\left(\frac{\xi}{B}\right)^{\frac{m_{m}^{0}+m_{s}^{0}}{2}}}{\sqrt{\pi} \Gamma\left(m_{m}^{0}\right) \Gamma\left(m_{s}^{0}\right)} \\
& \times G_{2,3}^{2,2}\left[\frac{\xi}{B} \mid \begin{array}{l}
1-\left(m_{m}^{0}+m_{s}^{0}\right) / 2,-\left(m_{m}^{0}+m_{s}^{0}-1\right) / 2 \\
\left(m_{s}^{0}-m_{m}^{0}\right) / 2,\left(m_{m}^{0}-m_{s}^{0}\right) / 2,-\left(m_{m}^{0}+m_{s}^{0}\right) / 2
\end{array}\right]
\end{aligned}
$$

where $G[\cdot]$, is the Meijer's G-function [24].

\section{Performance of the Cooperative System}

\subsection{Outage Probability}

In the cooperative case, the outage probability $P_{\text {out }}$ is obtained by the contribution of all the relay nodes belonging to the set $Q$ and the direct link using the MRC (Maximum Ratio Combining) technique. Thus, by using the law of total probability we obtain

$$
\begin{aligned}
P_{\text {out }} & =\sum_{Q} P\left(\gamma_{D}<\gamma_{D}^{\text {th }} \mid Q\right) P(Q) \\
& =\sum_{\bar{J}=0}^{J} P\left(\gamma_{D}<\gamma_{D}^{\text {th }}|| Q \mid=\bar{J}\right) P(|Q|=\bar{J})
\end{aligned}
$$

where $P(|Q|=\bar{J})$ is the probability that $\bar{J}$ nodes cooperate in relaying information to the destination.

$P\left(\gamma_{D}<\gamma_{D}^{\text {th }} \| Q \mid=\bar{J}\right)$ is the conditional outage probability in the case of $|Q|=\bar{J}$. In the following, we shall derive the expression of $P(|Q|=\bar{J})$ and $P\left(\gamma_{D}<\gamma_{D}^{t h}|| Q \mid=\bar{J}\right)$, respectively.

a) Calculation of $P(|Q|=\bar{J})$

The probability that the $j$-th relay-node is in the decoding set $Q$, is defined as the probability that its SNR, $\tilde{\gamma}_{j}$, is larger than the threshold SNR at the $j$-th relay, $\tilde{\gamma}_{j}^{\text {th }}$. It is given as follows

$$
A_{j}=P\left(\tilde{\gamma}_{j}>\tilde{\gamma}_{j}^{t h}\right), \quad j \in[1, J]
$$

For simplicity, we choose the same threshold for all the relays, i.e, $\tilde{\gamma}_{j}^{\text {th }}=\tilde{\gamma}^{\text {th }}$.

Consequently, $A_{j}$ is given as follows

$$
A_{j}=\int_{\tilde{\gamma}^{h h}}^{+\infty} p_{\tilde{\gamma}_{j}}(\gamma) \mathrm{d} \gamma=1-\int_{0}^{\tilde{\gamma}^{h h}} p_{\tilde{\gamma}_{j}}(\gamma) \mathrm{d} \gamma,
$$

where $p_{\tilde{\gamma}_{j}}(\gamma)$ is the probability density of the SNR at the $j$-th relay. It is given by (6) and its cdf function is given by (16) by setting $\xi_{j}=\frac{\tilde{m}_{m}^{j} \tilde{m}_{s}^{j}}{\tilde{\gamma}_{j}}$. Thus, $A_{j}$ is deduced easily.

Let $\Phi(\bar{J})$ denotes a combination that $\bar{J}$ of $J$ nodes cooperate in relaying information to the destination and $P_{\Phi(\bar{J})}$ as the corresponding probability. Assuming that the relay channels are independent, $P_{\Phi(\bar{J})}$ can be expressed as

$$
P_{\Phi(\bar{J})}=\prod_{j=1}^{\bar{J}} A_{j} \prod_{j^{\prime}=1, j^{\prime} \in \notin \Phi(\bar{J})}^{J}\left(1-A_{j^{\prime}}\right) .
$$

Consequently,

$$
P(|Q|=\bar{J})=\sum_{\Phi(\bar{J})} P_{\Phi(\bar{J})} .
$$

b) Calculation of $P\left(\gamma_{D}<\gamma_{D}^{\text {th }}|| Q \mid=\bar{J}\right)$ $P\left(\gamma_{D}<\gamma_{D}^{t h} \| Q \mid=\bar{J}\right)$ can be seen as the cdf of $\gamma_{D}=\sum_{j=0}^{\bar{J}} \gamma_{j}$, evaluated at $\gamma_{D}^{\text {th }}$, i.e. the sum of the $\bar{J}+1$. Generalized-K random variables correspond to the $\bar{J}+1$ active links. As mentioned above in Section 2.4, to avoid analytical complexity, the computation of the cdf expression of $\gamma_{D}$ can be approximated by the Gamma pdf with the scale and shape parameters given by (11) and (12), respectively. Consequently, $\gamma_{D} \sim \Gamma\left(\tilde{\theta}_{\bar{J}+1}, \tilde{k}_{\bar{J}+1}\right)$ and it is expressed as

$$
p_{\gamma_{D}}(\gamma)=\frac{1}{\Gamma\left(\tilde{k}_{\bar{J}+1}\right)}\left(\frac{\tilde{k}_{\bar{J}+1}}{\bar{\gamma}_{\bar{J}+1}}\right)^{\tilde{k}_{\bar{J}+1}} \gamma^{\tilde{k}_{\bar{J}+1}-1} \mathrm{e}^{-\frac{\tilde{\gamma}_{\bar{J}+1}}{\bar{\gamma}_{\bar{J}+1}}}
$$

where $\bar{\gamma}_{\bar{J}+1}=\tilde{\theta}_{\bar{J}+1} \frac{E s}{N 0}$.

Consequently $P\left(\gamma_{D}<\gamma_{D}^{t h} \| Q \mid=\bar{J}\right)$ is given as follows

$$
\begin{aligned}
& P\left(\gamma_{D}<\gamma_{D}^{t h} \| Q \mid=\bar{J}\right) \\
= & \int_{0}^{\gamma_{D}^{t h}} p_{\gamma_{D}}(\gamma) \mathrm{d} \gamma=\frac{\gamma_{i n c}\left(\tilde{k}_{\bar{J}+1}, \frac{\tilde{k}_{\bar{J}+1}}{\tilde{\theta}_{\bar{J}+1}} \gamma_{D}^{t h}\right)}{\Gamma\left(\tilde{k}_{\bar{J}+1}\right)},
\end{aligned}
$$

where $\gamma_{\text {inc }}(\cdot, \cdot)$ is the incomplete Gamma function. Finally, from (21)-(23) and (25) we derive the outage probability at the destination for the cooperative system as

$$
P_{\text {out }}=\sum_{\bar{J}=0 \Phi(\bar{J})}^{J} \sum_{j=1}^{\bar{J}} A_{j} \prod_{j^{\prime}=1, j^{\prime} \notin \Phi(\bar{J})}^{J}\left(1-A_{j^{\prime}}\right) \frac{\gamma_{\text {inc }}\left(\tilde{k}_{\bar{J}+1}, \frac{\tilde{k}_{\bar{J}+1}}{\tilde{\theta}_{\bar{J}+1}^{D}} \gamma_{\text {th }}^{D}\right)}{\Gamma\left(\tilde{k}_{\bar{J}+1}\right)}
$$

\subsection{Average Symbol Error Probability}

In the cooperative case, the ASEP $P_{s e}$ is obtained by the contribution of all the relay nodes and the direct link. At the MRC output, by considering the same notation as above, $P_{s e}$ can be expressed as follows

$$
P_{s e}=\sum_{Q} P_{s e}(Q) P(Q)=\sum_{J=0}^{J} P_{s e}(|Q|=\bar{J}) P(|Q|=\bar{J})
$$

$$
P(|Q|=\bar{J}) \text { has been calculated in the last subsection }
$$


given by (23). $P_{s e}(|Q|=\bar{J})$ is the conditional symbol error probability corresponding to the cooperation of the $\bar{J}$ branches and the direct link (source-destination). In the following, we shall derive its expression.

a) Calculation of $P_{s e}(|Q|=\bar{J})$

$P_{s e}(|Q|=\bar{J})$ can be seen as the probability of occurring an error when $\bar{J}$ relays are active. As mentioned in the last section, it can be expressed as

$$
P_{s e}(|Q|=\bar{J})=\int_{0}^{+\infty} P_{s e}\left(\gamma_{D}\right) p_{\gamma_{D}}(\gamma) \mathrm{d} \gamma,
$$

where $p_{\gamma_{D}}(\gamma)$ is given by (24) and $P_{s e}(|Q|=\bar{J})$ is expressed as

$$
P_{s e}(|Q|=\bar{J})=A \int_{0}^{+\infty} \operatorname{erfc}(\sqrt{B \gamma}) p_{\gamma_{D}}(\gamma) \mathrm{d} \gamma .
$$

To evaluate this integral, we use the relationship $Q(x)=\frac{1}{2} \operatorname{erfc}\left(\frac{x}{\sqrt{2}}\right)$ which leads to a new integral form as follows

$$
P_{s e}(|Q|=\bar{J})=2 A \int_{0}^{+\infty} Q(a \sqrt{\gamma}) p_{\gamma_{D}}(\gamma) \mathrm{d} \gamma,
$$

where $a=\sqrt{2 B}$. Using ([6], (5.1), (5.18)), this integral is deduced as follows.

For integer values of $\tilde{k}_{\bar{J}+1}, P_{s e}(|Q|=\bar{J})$ can be written as

$$
\begin{aligned}
& P_{s e}(|Q|=\bar{J}) \\
& =A\left[1-\sqrt{\frac{B \bar{\gamma}_{\bar{J}+1}}{\tilde{k}_{\bar{J}+1}+B \bar{\gamma}_{\bar{J}+1}}} \times \sum_{k=0}^{\tilde{k}_{J+1}}\left(\begin{array}{c}
2 k \\
k
\end{array}\right)\left(\frac{1}{4}-\frac{B \bar{\gamma}_{\bar{J}+1}}{4 \tilde{k}_{\bar{J}+1}+4 B \bar{\gamma}_{\bar{J}+1}}\right)^{k}\right] .
\end{aligned}
$$

For non-integer values of $\tilde{k}_{\bar{J}+1}, P_{s e}(|Q|=\bar{J})$ can be written as

$$
\begin{gathered}
P_{s e}(|Q|=\bar{J})=A \cdot C \frac{\sqrt{B \bar{\gamma}_{\bar{J}+1} / \tilde{k}_{\bar{J}+1}}}{\left(1+B \bar{\gamma}_{\bar{J}+1} / \tilde{k}_{\bar{J}+1}\right)^{\tilde{k}_{\bar{J}+1}+\frac{1}{2}}} \\
\times_{2} F_{1}\left(1, \tilde{k}_{\bar{J}+1}+\frac{1}{2}, \tilde{k}_{\bar{J}+1}+1, \frac{1}{1+\frac{B \bar{\gamma}_{\bar{J}+1}}{\tilde{k}_{\bar{J}+1}}}\right) .
\end{gathered}
$$

where $C=\Gamma\left(\tilde{k}_{\bar{J}+1}+1 / 2\right) /\left(\Gamma\left(\tilde{k}_{\bar{J}+1}+1\right) \sqrt{\pi}\right)$.

Thus, using (23), (27), (31) or (32), the ASEP $P_{s e}$ can be deduced easily.

\section{Simulation Results}

In this section, we present Monte-Carlo simulations to illustrate the previous theoretical results for both the outage probability and the ASEP.

\subsection{Outage Probability}

To be more accurate in our illustrations, we have simulated the shadowed Nakagami channel by taking into account the Nakagami-m distribution and the Log-normal distribution. For the non cooperative system, Figure 1 depicts the outage probability versus the threshold SNR, $\gamma_{D}^{\text {th }}$, for a transmit SNR of $E s / N 0=12 \mathrm{~dB}$. The Threshold SNR at the relays is chosen $\tilde{\gamma}^{\text {th }}=\gamma_{D}^{\text {th }}$. We have considered three different environments with increasing propagation hardness:

- Generalized-K channel with $m_{m}=1$ and $m_{s}=50$ : for high values of $m_{s}$, greater than 50 , we consider that there is no shadowing. It is equivalent to Rayleigh channel. The corresponding plots in Figure 1, show less outage probability compared to the others configurations. Moreover, they are close each other. We note the robustness of the channel simulation compared to the theoretical rayleigh performance. Besides, we deduce the accuracy of the approximated model compared to the exact formula;

- Generalized-K channel with $m_{m}=1$ and $m_{s}=2$;

- Generalized-K channel with $m_{m}=0.5$ and $m_{s}=2$.

For the two last configurations, we note similar performances between the Generalized-K cdf and the approximated Gamma cdf with an optimal value of $\varepsilon=1.6$ for $m_{m}=0.5$, and $\varepsilon=1$ for $m_{m}=1$. More precisely, the optimal value of $\varepsilon$ is obtained for lower tail approximation, since we are interested in small threshold values. We note just a small difference between theoretical results and simulation which is due to the approximation of the Log-normal pdf distribution by a Gamma distribution to model the shadowing fading.

In some cases, it is important to illustrate the outage

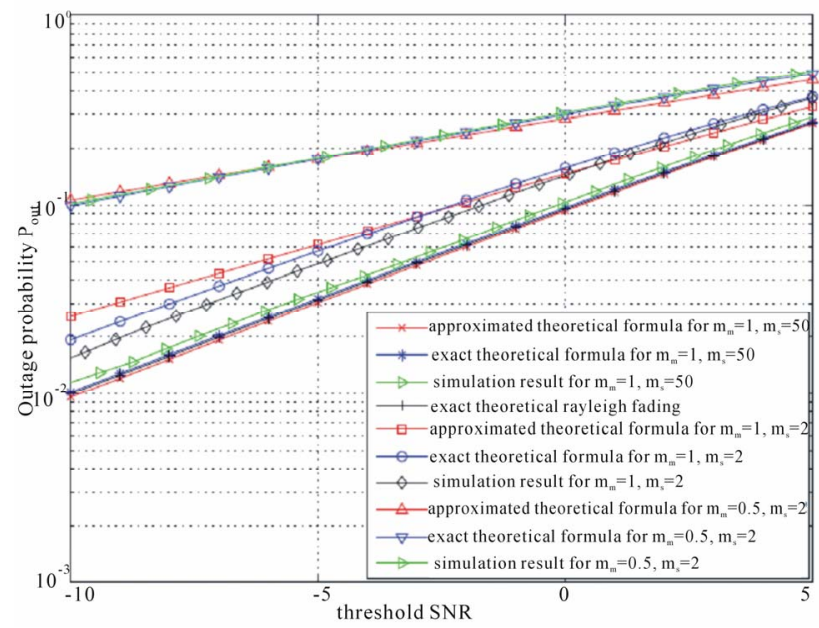

Figure 1. Outage probability versus the threshold SNR $\gamma_{D}^{\text {th }}$ for the non cooperative system. 
probability measure as function of the average SNR for a given threshold SNR. Figure 2 shows this illustration for $\gamma_{D}^{\text {th }}=5 \mathrm{~dB}$ for the same parameters of the three configurations mentioned above.

In the cooperative communication scenario, we have omitted the propagation environment relative to Rayleigh case; we have considered the two others environments cases corresponding to $m_{m}=1, m_{s}=2$ and $m_{m}=0.5$, $m_{s}=2$. For both cases, we have considered identical Nakagami-m shadowed fading channels for the links of the first hop and those of the second dual hop.

We have considered a number of relay nodes, $J=1,2$, and 3. Similarly to the non cooperative system, we have illustrated:

- The outage probability versus the threshold SNR $\gamma_{D}^{\text {th }}$ for a transmit SNR value of $E s / N 0=12 \mathrm{~dB}$ for both the first and the second hops. Figures 3 and $\mathbf{4}$ show

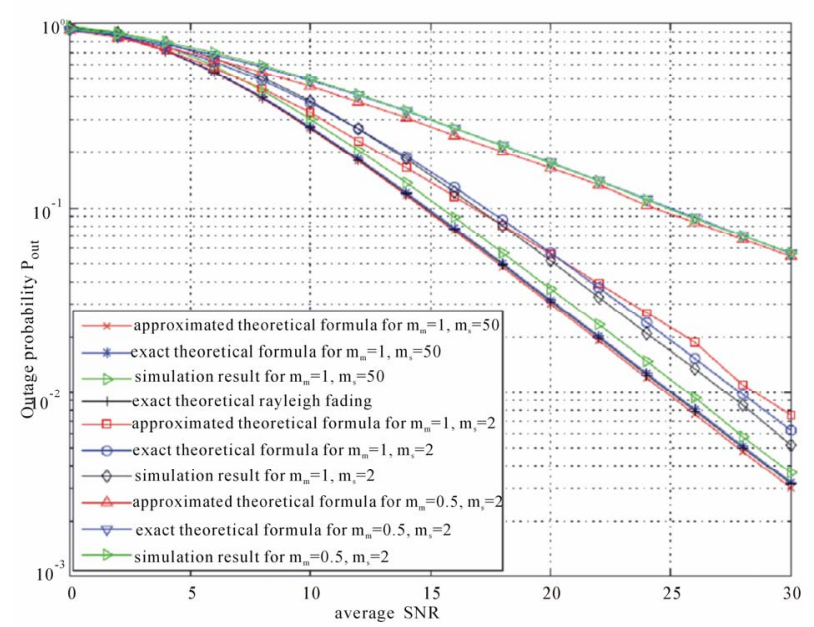

Figure 2. Outage probability versus $E s / N 0$ for the non cooperative system.

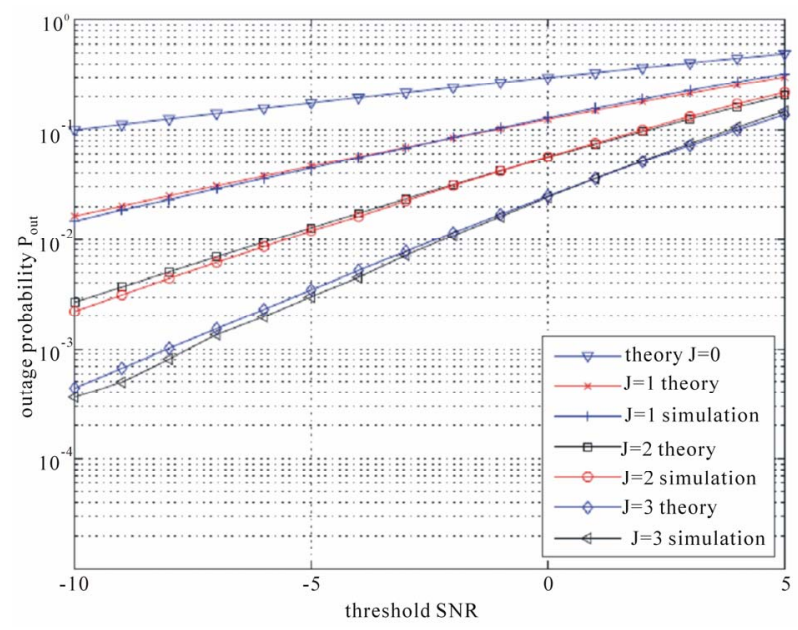

Figure 3. Outage probability versus the threshold SNR $\gamma_{D}^{\text {th }}$ for the cooperative system for $m_{m}=0.5, m_{s}=2$. the obtained results.

- The outage probability versus $E S / N 0$ for a given threshold SNR $\gamma_{D}^{\text {th }}=12 \mathrm{~dB}$. Figures 5 and $\mathbf{6}$ show the obtained results.

We note that, since we consider i.i.d channels, the optimal value of $\varepsilon$ is the same for all links, as mentioned in the theoretical analysis. Thus, $\varepsilon=1$ for $m_{m}=1$, $m_{s}=2$ and $\varepsilon=1.6$ for $m_{m}=0.5, m_{s}=2$. We observe that theoretical results match well simulation results. The little difference is again due to the approximation of the Log-normal distribution by a Gamma distribution. As expected, the use of more relays provides better outage performance.

\subsection{Average Symbol Error Probability}

In this subsection, we have illustrated the results relative

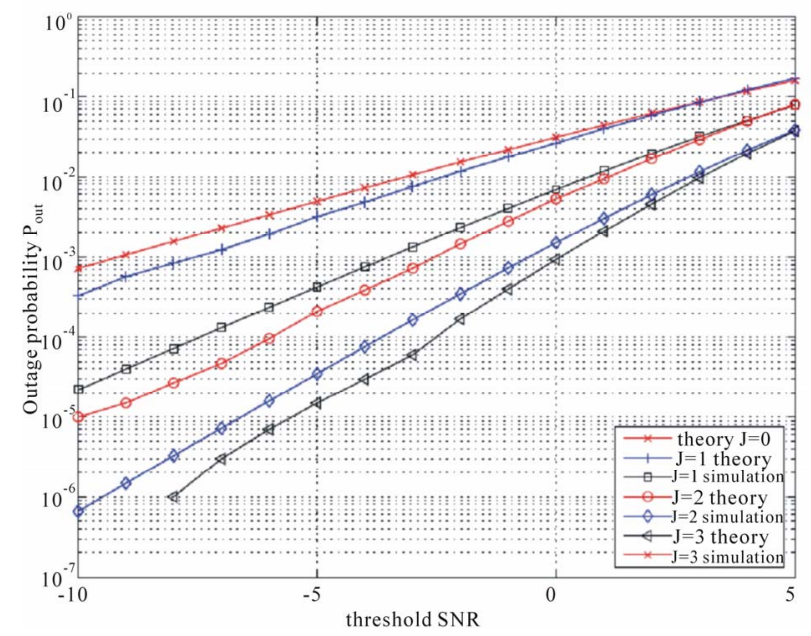

Figure 4. Outage probability versus the threshold SNR $\gamma_{D}^{\text {th }}$ for the cooperative system for $m_{m}=1, m_{s}=2$.

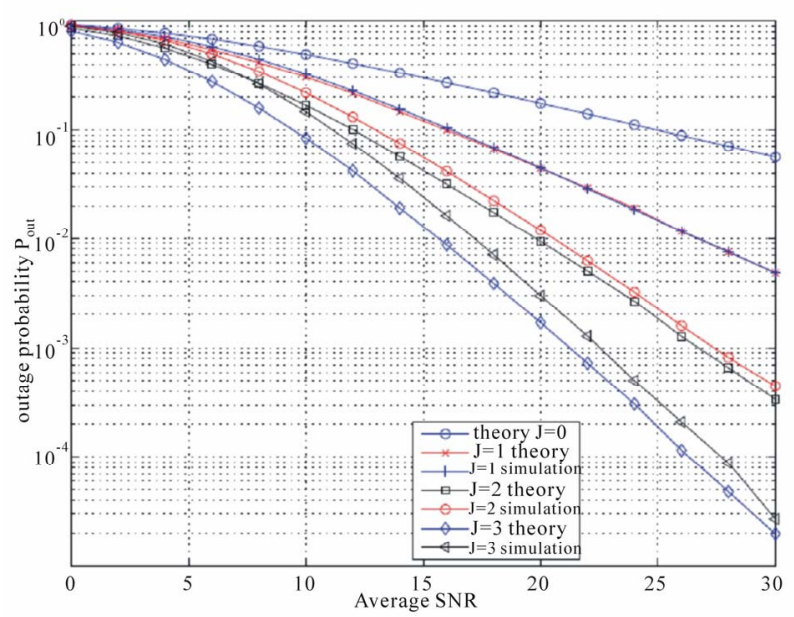

Figure 5. Outage probability versus $E s / N O$ for the cooperative system for $m_{m}=0.5$ and $m_{s}=2$. 
to the ASEP for 16-QAM modulated signals. We plot the performance curves in terms of the analytical and simulation ASEP versus $E s / N 0$ in dB. Similarly to the outage analysis, we consider two scenarii: the non cooperative and the cooperative systems. Figure 7 shows the obtained results for the first scenario.

We note a similar performance between the theoretical Rayleigh curve, the approximated results and the simulation results.

For the second scenario, we have considered identical and independent channels with the following parameters $m_{s}=2$ and $m_{m}=1$. We have considered a threshold SNR $\gamma_{D}^{\text {th }}=\tilde{\gamma}^{\text {th }}=15 \mathrm{~dB}$. To capture the path-loss effects, we have considered the following distances $d_{S, R}=0.7$, $d_{R_{j}, D}=0.8$ and $d_{S, D}=1$. We have chosen a number of relay nodes, $J=1,2$, and 3 . Obtained results are depicted in Figure 8. Optimal values of $\varepsilon$ are calculated for each $E s / N 0$ value. They are given by Table 1.

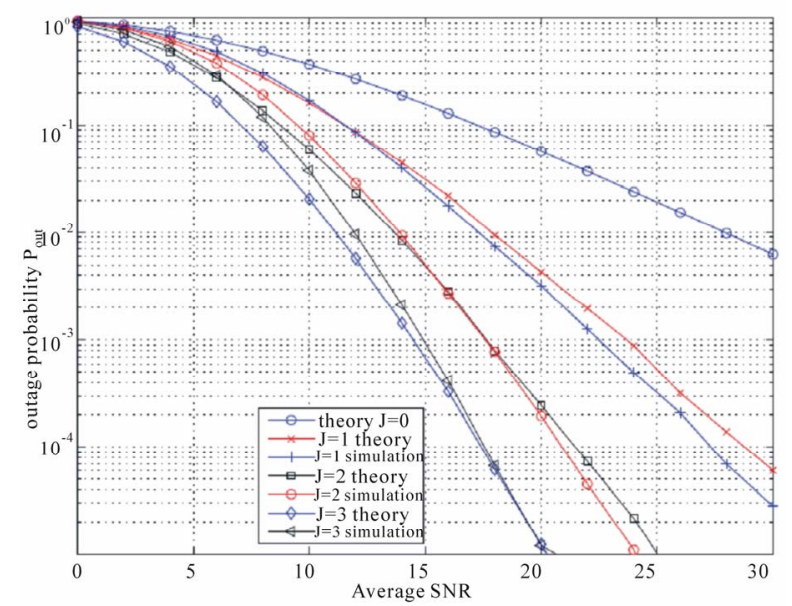

Figure 6. Outage probability versus $E s / N 0$ for the cooperative system for $m_{m}=1$ and $m_{s}=2$.

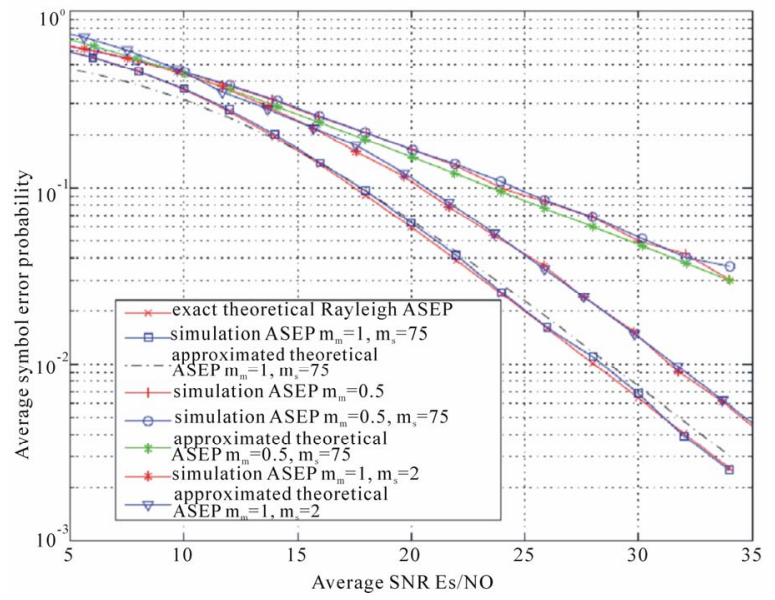

Figure 7. Average symbol error probability versus Es/N0 for the non cooperative system.

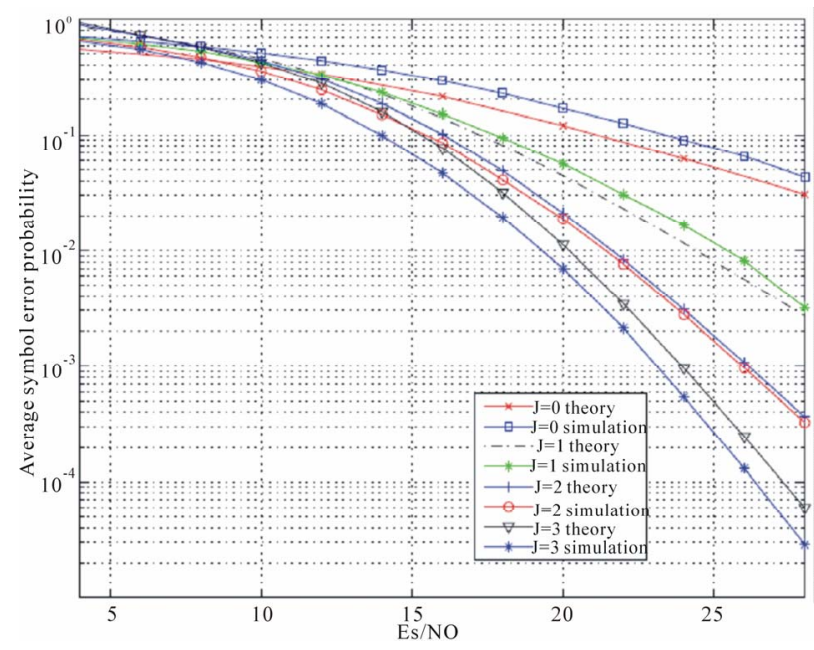

Figure 8. Average symbol error probability versus $E s / N 0$ for $m_{m}=1$ and $m_{s}=2$.

Table 1. $\varepsilon_{\text {opt }}$ versus Es/N0.

\begin{tabular}{|c|c|c|c|c|c|c|c|}
\hline$E s / N 0$ & 4 & 6 & 8 & 10 & 12 & 14 & 16 \\
\hline$\varepsilon_{o p t}$ & 0.67 & 0.69 & 0.7 & 0.71 & 0.72 & 0.73 & 0.74 \\
\hline \multicolumn{2}{|c|}{$E s / N 0$} & 18 & 20 & 22 & 24 & 26 & 28 \\
\hline \multicolumn{2}{|c|}{$\varepsilon_{o p t}$} & 0.74 & 0.75 & 0.75 & 0.76 & 0.76 & 0.76 \\
\hline
\end{tabular}

We note a good match between the simulation and theoretical results. The small difference between theoretical results and simulation includes the combination of the two approximations: approximation of the Lognormal pdf distribution by a Gamma distribution and the approximation of the Generalized-K distribution by a Gamma distribution. As expected, the use of more relays provides better ASEP performance.

\section{Conclusion}

We have analyzed the outage probability and the ASEP of an adaptive DF relaying over the composite fading multipath/shadowing channels modeled by a Generalized-K distribution. The utilization of an approximation of the Generalized-K pdf by an adjustable form of a Gamma distribution based on the two first moments matching method has been presented and validated by simulation under severe fading conditions. Based on this approximation, we derived closed-form expressions for the outage probability and the ASEP. We have provided Monte-Carlo simulations which are in good agreement with our theoretical analysis. This solution is helpful to investigate the performance analysis of cooperative networks under different environment conditions. 


\section{REFERENCES}

[1] A. Nosratinia, T. E. Hunter and A. Hedayat, "Cooperative Communication in Wireless Networks," IEEE Communications Magazine, Vol. 42, No. 10, 2004, pp. 74-80. doi:10.1109/MCOM.2004.1341264

[2] J. N. Laneman, D. N. C. Tse and G. W. Wornell, "Cooperative Diversity in Wireless Networks: Efficient Protocols and Outage Behavior," IEEE Transactions on Information Theory, Vol. 50, No. 12, 2004, pp. 3062-3080. doi:10.1109/TIT.2004.838089

[3] M. O. Hasna and M. S. Alouini, "Outage Probability of Multihop Transmission over Nakagami Fading Channels," IEEE Communications Letters, Vol. 7, No. 5, 2003, pp. 216-218. doi:10.1109/LCOMM.2003.812178

[4] M. O. Hasna and M.-S. Alouini, "End-to-End Performance of Transmission Systems with Relays over Rayleigh-Fading Channels," IEEE Transactions on Information Theory, Vol. 1, No. 6, 2003, pp. 1126-1131.

[5] E. Zimmermann, P. Herhold and G. Fettweis, "On the Performance of Cooperative Relaying Protocols in Wireless Networks," Special Issue on European Wireless, Vol. 16, No. 1, 2005, pp. 5-16.

[6] M. K. Simon and M. S. Alouini, "Digital Communications over Fading Channels," 2nd Edition, Wiley-IEEE Press, Hoboken, 2004. doi:10.1002/0471715220

[7] A. J. Coulson, A. G. Williamson and R. G. Vaughan, "A Statistical Basis for Lognormal Shadowing Effects in Multipath Fading Channels," IEEE Transactions on Communications, Vol. 46, No. 4, 1998, pp. 494-502. doi:10.1109/26.664305

[8] A. Abdi and M. Kaveh, "K-Distribution: An Appropriate Substitute for Rayleigh-Lognormal Distribution in Fading-Shadowing Wireless Channels," IEEE Electron Device Letters, Vol. 34, No. 9, 1998, pp. 851-852. doi:10.1049/el:19980625

[9] F. Hansen and F. Meno, "Mobile Fading-Rayleigh and Lognormal Superimposed," IEEE Transactions on Vehicular Technology, Vol. 26, No. 4, 1977, pp. 332-335. doi:10.1109/T-VT.1977.23703

[10] P. S. Bithas, N. C. Sagias, P. T. Mathiopoulos, G. K. Karagiannidis and A. A. Rontogiannis, "On the Performance Analysis of Digital Communications over Generalized-K Fading Channels," IEEE Communications Letters, Vol. 10, No. 5, 2006, pp. 353-355. doi:10.1109/LCOMM.2006.1633320

[11] P. S. Bithas, P. T. Mathiopoulos and S. A. Kotsopoulos, "Diversity Reception over Generalized-K (KG) Fading Channels," IEEE Transactions on Wireless Communications, Vol. 6, No. 12, 2007, pp. 4238-4243. doi:10.1109/TWC.2007.4400789

[12] N. C. Beaulieu and J. Hu, "A Closed-Form Expression for the Outage Probability of Decode-and-Forward Relaying Dissimilar Rayleigh Fading Channels," IEEE Communications Letters, Vol. 10, No. 12, 2006, pp. 813-815. doi:10.1109/LCOMM.2006.061048

[13] J. Hu and N. C. Beaulieu, "Closed-Form Expressions for the Outage and Error Probabilities of Decode-and-Forward Relaying in Dissimilar Rayleigh Fading Channels," IEEE International Conference on Communication, Glasgow, 24-28 June 2007, pp. 5553-5557.

[14] H. A. Suraweera, P. Smith and J. Armstrong, "Outage Probability of Cooperative Relay Networks in Nakagamim Fading Channels," IEEE Communications Letters, Vol. 10, No. 12, 2006, pp. 834-836. doi:10.1109/LCOMM.2006.060834

[15] C. K. Datsikas, N. C. Sagias, F. I. Lazarakis, and G. S. Tombras, "Outage Analysis of Decode and Forward Relaying over Nakagfami-m in Fading Channels," IEEE Signal Processing Letters, Vol. 15, 2008, p. 4144.

[16] S. S. Ikki and M. H. Ahmed, "Performance Analysis of Multi-Branch Decode-and-Forward Cooperative Diversity Networks over Nakagami-m Fading Channels," IEEE International Conference on Communications, St. John's, 14-18 June 2009, pp. 1-6.

[17] S. Ikki and M. H. Ahmed, "Performance Analysis of Cooperative Diversity Wireless Networks over Nakagami-m Channels," IEEE Communications Letters, Vol. 11, No. 4, 2007, pp. 334-336. doi:10.1109/LCOM.2007.348292

[18] G. P. Efthymoglou, N. Bissias and V. A. Aalo, "On the Error Rate Analysis of Dual-Hop Amplify-and-Forward Relaying in Generalized-K Fading Channels," Journal of Electrical and Computer Engineering, 2010, pp. 1-5. doi: $10.1155 / 2010 / 584594$

[19] A. Dziri, D. L. Ruyet, D. Roviras and M. Terre, "Outage Probability Analysis of the Decode and Forward Relaying over the Composite Fading Multipath/Shadowing Channels," 2010 IEEE Eleventh International Workshop on Signal Processing Advances in Wireless Communications (SPAWC), Paris, 20-23 June 2010, pp. 1-5. doi:10.1109/SPAWC.2010.5670907

[20] A. Dziri, D. Le Ruyet, D. Roviras and M. Terré, "Symbol Error Probability Analysis of the Decode and Forward Relaying over the Composite Fading Multipath/Shadowing Channels," IEEE International Symposium on Signal Processing and Information Technology, Luxor, 2010, pp. 171-176. doi:10.1109/ISSPIT.2010.5711768

[21] I. Kostic, “Analytical Approach to Performance Analysis for Channel Subject to Shadowing and Fading," IEEE Proceedings-Communications, Vol. 152, No. 6, 2005, pp. 821-827.

[22] S. Al-Ahmadi and H. Yanikomeroglu, "On the Approximation of the Generalized-K pdf by a Gamma pdf Using the Moment Matching Method," IEEE Wireless Communications and Networking Conference, Ottawa, 5-8 April 2009, pp. 1-6.

[23] M. Gu and D. A. Abraham, "Using McDaniels Model to Represent Non-Rayleigh Reverberation," IEEE Transactions on Oceanic Engineering, Vol. 26, No. 3, 2001, pp. 348-357.

[24] I. S. Gradshteyn and I. M. Ryzhik, "Table of Integrals, Series, and Products," 6th Edition, Academic Press, New York, 2000. 